\title{
NOT ALL LINKS ARE CONCORDANT TO BOUNDARY LINKS
}

\author{
T. D. COCHRAN AND K. E. ORR
}

\section{INTRODUCTION}

A link is a smooth, oriented submanifold $L=\left\{K_{1}, \ldots, K_{m}\right\}$ of $S^{n+2}$ which is the ordered disjoint union of $m$ manifolds each piecewise-linearly homeomorphic to the $n$-sphere (if $m=1, L$ is called a knot). Knots and links play an essential role in the classification of manifolds and, in this regard, perhaps the most important equivalence relation on links is that of link concordance. $L_{0}$ and $L_{1}$ are concordant if there is a smooth, oriented submanifold $C=\left\{C_{1}, \ldots, C_{m}\right\}$ of $S^{n+2} \times[0,1]$ which meets the boundary transversely in $\partial C$, is piecewise-linearly homeomorphic to $L_{0} \times[0,1]$ and meets $S^{n+2} \times\{i\}$ in $L_{i}$ for $i=0,1$. The particular situation which led to the introduction of this equivalence relation and which indicates its importance is as follows. If $S$ is an immersed 2-disk or 2-sphere in a 4-manifold $X, x_{0}$ is a singular value and $B$ is a small 4-ball neighborhood of $x_{0}$, then $S \cap B$ is a link in $S^{3}$. If $L$ were concordant to a link whose components bound disjoint 2-disks in $S^{3}$ (the latter is called a trivial link) then the singularity at $x_{0}$ could be removed. Thus the fundamental problem is to classify (for fixed $m, n$ ) the set of concordance classes.

In the mid-1960s M. Kervaire and J. Levine gave an algebraic classification of the high-dimensional $(n>1)$ knot concordance groups [L2]. For even $n$ these are the trivial group and for odd $n$ they are infinitely generated. In a sequence of papers S. Cappell

Received by the editors September 15, 1989 and, in revised form, December 5, 1989.

1980 Mathematics Subject Classification (1985 Revision). Primary 57Q45; Secondary 57M25, 57R67, 19G12.

Key words and phrases. Link, link cobordism, concordance, boundary link, homology, cobordism.

The first author was partially supported by National Science Foundation grant DMS-8903514.

The second author was partially supported by National Science Foundation Postdoctoral Fellowship. 
and J. L. Shaneson showed that these groups play the role of a natural coefficient system for the general study of piecewise-linear immersions and embeddings (see [CS2] for a survey). A computable classification of link concordance should prove equally enlightening. More recently, M. H. Freedman has shown that certain three-dimensional link concordance problems constitute a universal surgery problem in dimension four, and lie at the heart of the unresolved four-dimensional topological surgery conjecture [FQ]. Thus these problems are obstacles to classification results for topological 4-manifolds.

One serious dilemma has been the apparent lack of a group structure on the set of equivalence classes. For knots, the following "connected-sum" operation does induce an abelian group structure. Given knots $K$ and $K^{\prime}$ in $S^{n+2}$ separated by a hyperplane $P$, choose two embedded arcs which begin at $K$ (respectively, $K^{\prime}$ ), end at $P$ and are otherwise disjoint from $K \cup K^{\prime} \cup P$. The knot $K \sharp K^{\prime}$ is given by "tubing" $K$ to $K^{\prime}$ along the arcs. The obvious analogue for $m>1$ (choosing $m$ pairs of arcs) fails to be independent of the arcs.

Extending Levine's knot classification to links is also difficult because it is known that the fundamental group of the exterior of the link $\left(\pi_{1}\left(S^{n+2}-\left(L \times \stackrel{\circ}{D}^{2}\right)\right)\right)$ carries vital information, but these groups are large, typically containing non-abelian free subgroups. Moreover these groups are not invariant under concordance. The inclusion of a link exterior into a concordance exterior induces an isomorphism on integral homology but not on fundamental group.

However, the knot classification techniques do extend to a special class of links called boundary links. Recall that a Seifert manifold for a component $K_{i}$ of $L$ is a compact, oriented, connected submanifold of $S^{n+2}$ whose boundary is $K_{i}$. A boundary link is one which admits a collection of pairwise disjoint Seifert manifolds, one for each of its components. Since Kervaire and Levine relied heavily on Seifert manifolds, their techniques extend. Moreover, connected-sum can be used to define a group operation on this class of links. Cappell and Shaneson were the first to classify boundary links modulo a similarly restricted type of concordance. Their techniques of homology surgery suggested further progress, culminating in the work of J. Le Dimet (see §3) [CS1, LD].

The only heretofore realized obstructions to a links being concordant to a boundary link were Massey products in the link ex- 
terior (so called higher-order linking numbers) or, equivalently, Milnor's $\bar{\mu}$-invariants (see $[\mathrm{H}])$. These always vanish if $n>1$.

In this paper we give infinite classes of counterexamples to the long-standing conjecture that every higher-dimensional link is concordant to a boundary link and the analogous conjecture for the 3-sphere.

Theorem A. There are infinitely many concordance classes of links in $S^{2 n+1}$, (with vanishing Massey products if $\left.n=1\right)$, containing no links concordant to boundary links.

Among these are infinite families (e.g., Fig. 1) with the properties that framed surgery on the components of the link yields a manifold homeomorphic to framed surgery on a boundary link, and that (if $n>1$ ) $\pi_{1}$ of the link exterior is the free group of rank $m$. If arcs are chosen for each of these links, so that connected sum is well-defined, then these infinite families form linearly independent sets. This is to say that the family comes from an infinite linearly independent set of disk links (see §3). Note that we have no results for even-dimensional links.

A careful analysis of a result of Hillman along with the classification results of Le Dimet sparked our thinking [LD] [H; Thm. 5, p. 150]. The precise relationship between our work and Le Dimet's is given in $\S 3$. Our results were first obtained by these techniques. However $\S 1$ gives a presentation of our simplest invariants using only linking pairings on metacyclic covering spaces.

We are indebted to Jim Davis and Bruce Williams for their help with various aspects of $L$-theory.

\section{$\S 1$. SOME INVARIANTS}

If $L=\left(K_{1}, K_{2}\right)$ is a link in $S^{2 n+1}$, let $M$ denote the two-fold cover of $S^{2 n+1}$ branched over $K_{1}$, and $J=\left\{J_{1}, J_{2}\right\}$ denote the lifts of $K_{2}$ ordered arbitrarily. $J$ is a link in a $\mathbf{Z}_{(2)}$-homology sphere. We call this the covering link of $L$ and denote it by $\widetilde{L}$.

The covering link can be used to distinguish links from boundary links. If $L$ is a boundary link, then the meridians of $\widetilde{L}$ generate $H_{1}(M-\widetilde{L}) /$ torsion. However this is not true in general. In the following, $\mu_{i}$ is a meridian for $J_{i}$.

Lemma 1. There is a unique epimorphism $\rho: \pi_{1}(M-\widetilde{L}) \rightarrow \mathbf{Z}$ such that $\rho\left(\mu_{1}\right)=-\rho\left(\mu_{2}\right)$ and such that $\rho\left(\mu_{1}\right)$ is an odd positive 
integer. Reordering the components of the covering link does not change $\left|\rho\left(\mu_{1}\right)\right|$. If $L$ is a boundary link then $\rho\left(\mu_{1}\right)=1$.

Define the complexity of the link to be the number $\rho\left(\mu_{1}\right)$ given by this lemma.

Outline of the proof of Theorem A. First observe that if $L$ is concordant to $L^{\prime}$ by a concordance $\left(C_{1}, C_{2}\right)$, and if $M$ is the twofold branched cover of $C_{1}$, then $M$ is a $\mathbf{Z}_{(2)}$-homology $S^{2 n+1} \times[0,1]$, and $C_{2}$ lifts to a concordance between the covering links of $L$ and $L^{\prime}$. We define invariants of the concordance class of the covering link $\widetilde{L}$ of $L$ and, a fortiori, of the concordance class of $L$.

Let $(N, \lambda)$ be the Blanchfield pairing associated to the Z-cover. $\widetilde{M}$, of the exterior of the covering link of $L$ guaranteed by Lemma 1 . If $n=1$ then let $\widetilde{M}$ be the cover of zero surgery on $\widetilde{L}$ in $M$. $N=T_{n}^{S}(\widetilde{M})$, the $S$-torsion part of $H_{n}(\widetilde{M})$, and $\lambda$ is a $(-1)^{n+1}$. Hermitian linking pairing, $\lambda: T_{n}^{S}(\widetilde{M}) \times T_{n}^{S}(\widetilde{M}) \rightarrow S^{-1} \Lambda / \Lambda$, where $\Lambda=\mathbf{Z}\left[t, t^{-1}\right]$ and $S=1+\operatorname{ker}\left\{\varepsilon: \mathbf{Z}_{(2)}\left[t, t^{-1}\right] \rightarrow \mathbf{Z}_{(2)}\right\}$. For each odd integer $i$, let $W_{i}=L_{*}\left(\mathbf{Z}_{(2)}\left[t, t^{-1}\right], S\right) . W_{i}$ is the Witt group of linking forms on (homological dimension one), $S$ torsion $\mathbf{Z}_{(2)}\left[t, t^{-1}\right]$ modules. Let $\widehat{W}=L_{*}\left(\mathbf{Z}_{(2)}\left[\mathbf{Z}_{(2)}\right], T\right)$ where $T=1+\operatorname{ker}\left\{\varepsilon: \mathbf{Z}_{(2)}\left[\mathbf{Z}_{(2)}\right] \rightarrow \mathbf{Z}_{(2)}\right\}$. Define $\lambda(L)=(N, \lambda) \in W_{c(L)}$ and $\hat{\lambda}(L)$ to be the image of $\lambda(L)$ under the natural homomorphism $W_{c(L)} \rightarrow \widehat{W}$ defined by sending 1 to $1 / c(L) \in \mathbf{Z}_{(2)} \subset \mathbf{Q}$.

The complexity of a link is not a concordance invariant, so neither is $\lambda(L)$. However, one can define the complexity of a concordance. Furthermore, the complexity of the link divides the complexity of any concordance of the link. Using this observation, one shows that $\hat{\lambda}(L)$ is an invariant of the concordance class of $L$. By Lemma 1 , if $L$ is a boundary link then $\hat{\lambda}(L) \in \operatorname{Image}\left\{W_{1} \rightarrow\right.$ $\widehat{W}\}$.

To study $\hat{\lambda}(L) \in \widehat{W}$, we look at its image under the map

$$
L_{*}\left(\mathbf{Z}_{(2)}\left[\mathbf{Z}_{(2)}\right], T\right) \rightarrow L_{*}\left(\mathbf{Q}\left[\mathbf{Z}_{(2)}\right], T\right)=\lim _{\rightarrow} L_{*}\left(\mathbf{Q}\left[t, t^{-1}\right], S\right) .
$$

If $\bar{W}_{i}=L_{*}\left(\mathbf{Q}\left[t, t^{-1}\right], S\right)$, the map $\bar{W}_{i} \rightarrow \bar{W}_{j}$ in the above system, is induced from the map of rings sending $t$ to $t^{j / i}$. Since $\mathbf{Q}\left[t, t^{-1}\right]$ is a principle ideal domain, a form over $\mathbf{Q}\left[t, t^{-1}\right]$ can be decomposed into its primary parts. These parts, in turn, are equivalent to forms in the $L$-theory of number fields, and may be detected by signatures. A careful analysis proves this proposition. 
Proposition 2. $\widehat{W} / W$ contains an infinitely generated free summand.

It remains to calculate some examples. It happens that the above invariant is always zero if $n$ is even, so in this case invariants associated to three-fold branched covers must be used.

\section{§2. EXAMPLES}

We describe a construction of a family of homology boundary links $L=\left\{K_{1}, K_{2}\right\}$ in $S^{2 n+1}$ which realizes a broad range of elements of $W$ (independent of $c(L)$ ). These examples work for all $n$. Consider a knot $K$ in $S^{2 n+1}$ and an untwisted $I$-bundle over it as shown schematically in Figure 1. Consider the ambient plumbing with $S^{1} \times D^{2 n-1}$ whose core circle can wrap arbitrar$i l y$, algebraically linking the first band $m$ times. (The simplest example is shown in Figure 1.)

Let $K_{1}$ be the boundary of this plumbing and let $K_{2}$ be as shown. This is a homology boundary link (by [C]) with complexity $2 m+1$ whose Blanchfield pairing is independent of $m$ and is closely related to the ordinary pairing of $K$.

In the above examples, if $m \neq 0$ and $K$ is any representative of a non-torsion class in the subgroup of the algebraic knot concordance group, then the resulting link is not concordant to any

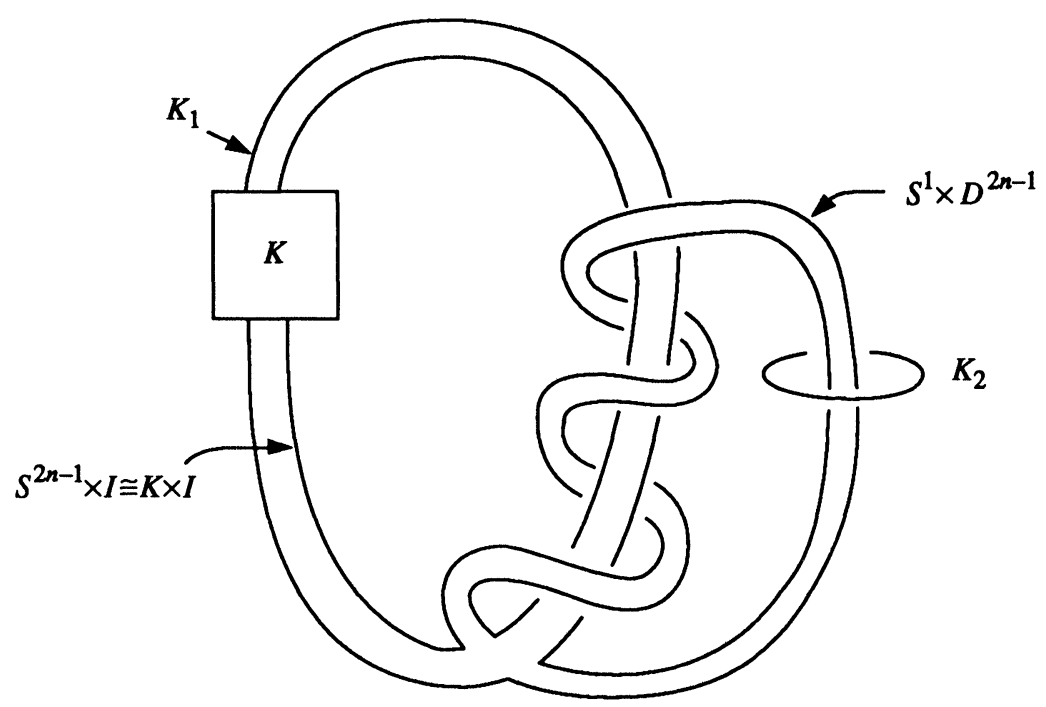

FIGURE 1 
boundary link. (Neither is it concordant to a link whose first component is unknotted!) Note that (zero framed for $n=1$ ) surgery on these links gives a manifold which does not depend on $m$. For $m=0$, this is surgery on a boundary link!

\section{§3. Relation to Le Dimet's work}

In [LD], Le Dimet classifies higher-dimensional $m$-component disk links up to disk link concordance. An $m$-component $n$ dimensional disk link is a piecewise linear locally flat embedding $\{1, \ldots, m\} \times D^{n} \rightarrow D^{n+2}$, transverse to the boundary and inducing a fixed trivial embedding on the boundary. Concordances are assumed to be constant on the boundary.

Consider the diagrams below where $\widehat{F}$ is the Vogel localization of the free group, $F$, on $m$ letters:

$$
\begin{array}{cccccc}
\mathbf{Z} F & \rightarrow & \mathbf{Z} F & \mathbf{Z} F & \rightarrow & \mathbf{Z} F \\
\downarrow & \mathbf{\Phi} & \downarrow & \downarrow & \Psi & \downarrow \\
\mathbf{Z} F & \rightarrow & \mathbf{Z} & \mathbf{Z} \widehat{F} & \rightarrow & \mathbf{Z} .
\end{array}
$$

Le Dimet defines a homotopy invariant and shows that a quotient of the Cappell-Shaneson homology surgery group $\Gamma_{n+3}(\Psi)$ classifies disk links with vanishing homotopy invariant. By CappellShaneson [CS1], boundary links (up to boundary link concordance) are classified by $\Gamma_{n+3}(\Phi)$. Thus, to find disk links not concordant to boundary links, it suffices to show that $\varphi: \Gamma_{n+3}(\mathbf{Z}[F] \rightarrow \mathbf{Z}) \rightarrow$ $\widetilde{\Gamma}_{n+3}(\mathbf{Z}[\widehat{F}] \rightarrow \mathbf{Z})$ is not onto where the target group is the appropriate quotient of $\Gamma_{n+3}(\mathbf{Z}[\widehat{F}] \rightarrow \mathbf{Z})$ (see [LD]).

We consider two component links and calculate the Vogel localization $\widehat{D}$ of the infinite dihedral group $D . \widehat{D}$ fits into the exact sequence $\mathbf{Z}_{(2)} \rightarrow \widehat{D} \rightarrow \mathbf{Z}_{2}$, where $\mathbf{Z}_{2}$ acts by negation on $\mathbf{Z}_{(2)}$, the integers localized at 2. Any epimorphism $\alpha: F \rightarrow D$ induces an epimorphism $\hat{\alpha}: \widehat{F} \rightarrow \widehat{D}$ so that, for $n$ odd, the cokernel of $\varphi$ maps onto the cokernel of $\psi: \Gamma_{n+3}(\mathbf{Z}[D] \rightarrow \mathbf{Z}) \rightarrow \Gamma_{n+3}(\mathbf{Z}[\widehat{D}] \rightarrow$ Z).

There are sequences of homomorphisms

$$
\Gamma_{*}(\mathbf{Z}[D] \rightarrow \mathbf{Z}) \rightarrow \Gamma_{*}\left(\mathbf{Z}_{(2)}[\mathbf{Z}] \rightarrow \mathbf{Z}_{(2)}\right) \rightarrow L_{*}\left(\mathbf{Z}_{(2)}[\mathbf{Z}], S\right)
$$

and

$$
\Gamma_{*}(\mathbf{Z}[\widehat{D}] \rightarrow \mathbf{Z}) \rightarrow \Gamma_{*}\left(\mathbf{Z}_{(2)}\left[\mathbf{Z}_{(2)}\right] \rightarrow \mathbf{Z}_{(2)}\right) \rightarrow L_{*}\left(\mathbf{Z}_{(2)}\left[\mathbf{Z}_{(2)}\right], T\right) .
$$

In these sequences, the first homomorphisms are transfers to the index two subgroups. The second homomorphisms take the associated Blanchfield pairings (see $[R]$ ). 
Theorem C. The invariant $\hat{\lambda}(L)$ defined in $\S 2$ is related to Le Dimet's surgery invariant via the composition

$$
\begin{aligned}
& \Gamma_{4 n}(\mathbf{Z}[\widehat{F}] \rightarrow \mathbf{Z}) \rightarrow \Gamma_{4 n}(\mathbf{Z}[\widehat{D}] \rightarrow \mathbf{Z}) \\
& \quad \rightarrow L_{4 n}\left(\mathbf{Z}_{(2)}\left[\mathbf{Z}_{(2)}\right], S\right) \rightarrow L^{4 n}\left(\mathbf{Z}_{(2)}\left[\mathbf{Z}_{(2)}\right], S\right) .
\end{aligned}
$$

Boundary links come from the analogous composition

$$
\begin{aligned}
& \Gamma_{4 n}(\mathbf{Z}[F] \rightarrow \mathbf{Z}) \rightarrow \Gamma_{4 n}(\mathbf{Z}[D] \rightarrow \mathbf{Z}) \\
& \quad \rightarrow L_{4 n}\left(\mathbf{Z}_{(2)}[\mathbf{Z}], T\right) \rightarrow L^{4 n}\left(\mathbf{Z}_{(2)}[\mathbf{Z}], T\right) .
\end{aligned}
$$

\section{FURTHER COMMENTS}

The above invariants and examples are special cases of very general techniques. Besides twofold covers, we consider $p^{r}$-fold covers for prime $p$, or even more general nilpotent covers. In all of these cases the covering link will have many components so we consider Blanchfield pairings associated to the maximal free Abelian cover. Each of these corresponds to constructing a nilpotent-byfree Abelian quotient of the free group (like the infinite dihedral group), localizing this group, and transferring between homology surgery groups to enable computation. If one can calculate without transferring, one accesses even more general invariants. More importantly, we can iterate this process, since $p^{r}$-fold branched covers of $\mathbf{Z}_{(p)}$-homology spheres are $\mathbf{Z}_{(p)}$-homology spheres. This leads to irregular covering spaces which detect information even when each component of $L$ is unknotted. There are many examples detected by iterated covers but not by any single cover. The breadth of these techniques gives hope that, with more work, one can calculate the algebraic disk link concordance group, classifying concordance of sublinks of homology boundary links (see [L, LMO]), and combined with the results of Cappell, Shaneson, and Le Dimet, classify all counterexamples to the conjecture, now known to be false, that all higher-dimensional links are concordant to boundary links.

\section{REFERENCES}

[CG1] A. Casson and C. McA. Gordon, Cobordism of classical knots (A. Marin and L. Gillou, eds.), preprint, Orsay (Reprinted in A la recherche de la Topologie perdue, Progr. Math. 62, Birkhauser, Boston, 1986.).

[CG2] A. Casson and C. McA. Gordon, On slice knots in dimension three, Proc. Sympos. Pure Math. 30 (1978), 39-53.

[C] T. D. Cochran, Link concordance invariants and homotopy theory, Invent. Math. 90 (1987), 635-645. 
[CS1] S. Cappell and J. L. Shaneson, Link cobordism, Comment. Math. Helv. 55 (1980), 20-49.

[CS2] S. Cappell and J. L. Shaneson, An introduction to embeddings, immersions and singularities in codimension two, Proc. Sympos. Pure Math. 32 (1978), Ann. of Math. 99 (1974), 277-348.

[FQ] M. H. Freedman and F. Quinn, Topology of four manifolds, preprint.

[H] J. Hillman, Alexander ideals of links, Springer-Verlag Lecture Notes in Math. 895 (1981).

[L] J. Levine, Link concordance and algebraic closure, II, Inventiones Mathematicae 96 (1989), 571-592.

[L2] J. Levine, Knot cobordism groups in codimension two, Comment. Math. Helv. 44 (1969), 229-244.

[LD] J. Le Dimet, Cobordisme d'Enlacement de Disques, Memoir de la Mathematique de France 32, 116, 1988.

[LMO] J. Levine, W. Mio and K. Orr, Links with vanishing homotopy invariant, Brandeis University, preprint.

[R] A. Ranicki, Exact sequences in the algebraic theory of surgery, Princeton Univ. Press, vol. 26, 1981.

NORTHWESTERN UNIVERSITY, EVANSTON, ILLINOIS 60201

University of Chicago, Chicago, Illinois 60637 and Indiana University, BLOOMINGTON, INDIANA 